Die Bestimmung geringster Mengen von Gesamtphosphor im Wasser von Binnenseen

(Beiträge zur chemischen Wasser- und Abwasseranalyse, Nr. 2)

Von M. Schmid und H. Ambühl 


\title{
Die Bestimmung geringster Mengen von Gesamtphosphor im Wasser von Binnenseen
}

(Beitrăge zur chemischen Wasser- und Abwasseranalyse, Nr. 2)

\author{
Von M. SсHмID und H. AMBÜHL
}

Eidg. Anstalt für Wasserversorgung, Abwasserreinigung und Gewässerschutz an der Eidg. Technischen Hochschule, Zürich, Direktion: Prof. Dr. O. Jaag

Manuskript eingegangen am 14. Dezember 1964

\section{Einleitung}

Um die Rolle der Pflanzennährstoffe im limnischen Geschehen voll zu erfassen und auch den Gehalt der Seen an solchen Stoffen zu kennen, genügt es nicht, wenn man diese Nährstoffe, insbesondere Stickstoff und Phosphor, nur in ihrer anorganischen Form kennt. Diese Form, Nitrat und Phosphat hauptsächlich, nimmt zwar im biologischen Haushalt eines Sees eine wichtige Stellung ein, die Kenntnis ihres quantitativen Vorhandenseins ist aber nur ein Teil des Ganzen, ein Bruchstück aus einem grösseren Kreislauf, insbesondere beim Phosphor. Während der Stickstoff, wie jede langfristige Seeuntersuchung zeigt, nach dem Absterben der Organismen, in welche er von dem im Wasser in Lösung vorhandenen anorganischen Depot her eingebaut worden war, in die Seetiefe verfrachtet wird und damit bis zur nächsten Zirkulation dem weiteren Kreislauf entzogen bleibt, wird zumindest ein Teil des Phosphors noch in der trophogenen Schicht in gelöster, organisch gebundener Form, als sogenannter "organischer Phosphor» wieder freigesetzt, durch Bakterientätigkeit zu anorganischem Phosphat verarbeitet [8] und steht so noch in der trophogenen Schicht bereits wieder als wirksamer Nährstoff zur Verfügung. Neuesten Untersuchungen zufolge [7] ist mit sehr kurzen "Umlaufzeiten» zu rechnen, welche während der Sommerstagnation in der Grössenordnung von Minuten liegen können. So erstaunlich diese Feststellung an sich ist und so folgenschwer ihre praktischen Konsequenzen sein dürften; sowenig erstaunt es, dass unter solchen Umständen ein minimaler, mit bisherigen Methoden kaum mehr bestimmbarer Gehalt an Phosphor im Seewasser eine sichtbare Algenproduktion in Gang zu halten vermag. Damit verliert aber auch die Feststellung des jeweils vorhandenen Gehaltes an anorganischem Phosphat im Seewasser an Bedeutung, bzw. sie allein vermag über die Nährstoffverhältnisse nicht Auskunft zu geben, sondern wird erst dann Aussagewert erhalten, wenn sie durch die beiden andern Zustandsformen des Phosphors, nämlich den gelösten, bzw. kolloiden Phosphor in organischer Bindung und den geformten, abfiltrierbaren, ebenfalls organisch gebundenen Phosphor ergänzt wird. Erst diese drei Fraktionen zusammen geben Aufschluss über die momentane 
Nährstoffsituation. Zwar stellen sie ihrerseits, um den erwähnten neuen Beobachtungen zu folgen, auch nur einen Momentanzustand eines sehr dynamischen Systems dar, dessen Kenntnis, und namentlich die Kenntnis der Umsatzzeit oder -geschwindigkeit, in jedem praktischen Fall erst die letzte und wichtigste Information über den jeweiligen Phosphorkreislauf liefern würde, doch ist die Kenntnis der statischen Phosphorgehalte zunächst die Grundlage für alles weitere.

In einer ersten Mitteilung haben wir uns mit der Bestimmung des Phosphations in Seewasser auseinandergesetzt [1] und festgestellt, dass es mit einer Modifikation der bekannten Molybdänblaumethode im Direktverfahren, also ohne Anreicherung, möglich ist, jeden beliebigen Gehalt bis hinunter auf $0,0 \mu \mathrm{g} / \mathrm{l} \mathrm{P}$ mit einer mittleren quadratischen Abweichung von $s= \pm 0,2 \mu \mathrm{g} / \mathrm{l} \mathrm{P}$ zu bestimmen. Diese Methode liefert interessante Ergebnisse, welche jedoch aus den oben erwähnten Gründen unvollständig bleiben, indem die vorhandenen Verfahren zur Mikrobestimmung des Gesamtphosphors selten genaue, reproduzierbare Werte lieferten. Um neue, verfeinerte Kenntnisse über den Phosphorhaushalt in Seen zu erlangen, musste vorerst eine Methode gefunden werden, welche gestattet, den Gesamtphosphorgehalt in Seewasser mit annähernd der gleichen Präzision zu bestimmen wie den Phosphatgehalt.

Die Verfasser sind Herrn Bruno RiBI für seine zuverlässige Mitarbeit bei der Ausführung der heiklen Analysen zu Dank verpflichtet.

\section{Literatur}

Damit sie mit der Molybdatmethode bestimmt werden können, müssen organische Phosphorverbindungen aufgeschlossen, das heisst in anorganisches Orthophosphat überführt werden. Dieser Prozess kann nach verschiedenen Verfahren durchgeführt werden, welche sich vor allem durch das Aufschlussmittel, meist ein Säuregemisch, dann aber auch durch Einzelheiten in der Behandlung der Proben unterscheiden. So weist OHLE [6] auf die störende Wirkung der Aufschlussmittel (Schwefelsäure, Salzsäure, Salpetersäure) auf die nachfolgende kolorimetrische Bestimmung hin und erwähnt besonders den hemmenden Einfluss zurückgebliebener unzerstörter Spuren von Wasserstoffsuperoxyd, das er als Oxydationsreagens benützt, auf die Entwicklung des Molybdänblaus. VAN BENEDEN [2] zeigt mit Hydrolyseversuchen, dass für die Umwandlung von Meta- in Orthophosphat mit Hilfe von Schwefelsäure und Wasserstoffsuperoxyd 30 min Reaktionszeit genügen. Während BörTCHER [3] nur mit Perchlorsäure aufschliesst und dabei darauf hinweist, dass bei hohen Temperaturen Verluste von Phosphor eintreten können, arbeiten BURTON und RILEY [4] zusätzlich noch mit Salpetersäure. Zur Verkürzung der Aufschlusszeit gibt KapPLıN [5] zur schwefelsauren Lösung Kaliumchlorat $\mathrm{KClO}_{3}$ zu.

Statt mit solchen «nassen» Aufschlussmethoden kann die Oxydation der P-Verbindungen auch mit dem "trockenen" Aufschluss erreicht werden. Hier wird die zu untersuchende Probe unter Zusatz von Salzsäure (zur Zerstörung der Karbonathärte) in der Platinschale zur Trockne gedampft und anschliessend verascht. Der Rückstand wird mit verdünnter Salzsäure aufgenommen und während einer halben Stunde auf dem Wasserbad digeriert. Anschliessend wird mit Ammoniak neutralisiert.

Unsere Untersuchungen haben nun gezeigt, dass es in Anlehnung an die Methode von OHLE [6] mit Hilfe von Schwefelsäure und Wasserstoffsuperoxyd als Oxydations- 
mittel, zusammen mit den Verfeinerungen unserer Phosphatbestimmungsmethode [1] möglich ist, mit einer Genauigkeit zu arbeiten, welche derjenigen der Phosphatbestimmung sehr nahe kommt.

\section{Eigene Erfahrungen mit bestehenden Methoden Die neue Modifikation}

1936 hat W. OHLE eine Methode zur Gesamtphosphorbestimmung beschrieben [6]: Die Wasserprobe wird in einem Kjeldahlkolben mit konzentrierter Schwefelsäure versetzt, das Wasser auf dem Sandbad abgedampft und bis zum Auftreten von $\mathrm{SO}_{3^{-}}$ Dämpfen weitererhitzt. Danach erfolgt eine $\mathrm{Zugabe}$ von $\mathrm{H}_{2} \mathrm{O}_{2}$ und anschliessend wird mehrmals stark abgeraucht, bis die Dämpfe lichter werden. Der Aufschluss wird dann mit Ammoniak auf pH 7,0 neutralisiert, worauf eine bestimmte Menge an $1 n$ Schwefelsäure zuzugeben ist. Wir haben uns anfänglich an diese Vorschrift gehalten, dann aber beobachtet, dass die Prozedur des Aufschliessens besondere Bedingungen verlangt, die zudem sehr genau einzuhalten sind. Je nach Temperatur und Dauer des Aufschlusses gehen zusammen mit den $\mathrm{SO}_{3}$-Dämpfen unkontrollierbare Mengen an Phosphor verloren. Aus diesem Grund verwenden wir nicht mehr gasbeheizte Aufschlussbrenner oder Sandbäder, sondern regulierbare, mit einem elektrischen Thermometer geeichte elektrische Infrarotbrenner, auf denen die Wasserproben bis zum ersten Auftreten von $\mathrm{SO}_{3}$-Dämpfen abgedampft werden. Wird dieser Moment verpasst und entwickeln sich, wie es in den üblichen Vorschriften sogar verlangt wird, starke Schwaden von $\mathrm{SO}_{3}$-Dämpfen, so treten mit ziemlicher Sicherheit Verluste ein. Das erste Auftreten der Dämpfe darf deshalb nicht übersehen werden, und die Aufschlusskolben sind sofort wegzustellen. Nach dem Erkalten wird Wasserstoffsuperoxyd (Perhydrol Merck) zugesetzt, worauf der eigentliche Aufschluss auf einem zweiten Infrabrenner, der so eingestellt ist, dass das Aufschlussgut eine Temperatur von ca. $260^{\circ} \mathrm{C}$ erhält, vollzogen wird. Nach einer halben Stunde ist die Lösung klar und farblos und das überschüssige Peroxyd weitgehend zerstört. Die nachfolgende Zugabe von etwas destilliertem Wasser und nochmaliges Abdampfen ist lediglich eine Sicherheitsmassnahme, um zu gewährleisten, dass die letzten Spuren von Peroxyd zersetzt sind.

Die Temperatureichung der Aufschlussbrenner: Der Aufschlusskolben enthält während der Prozedur maximal $1 \mathrm{ml}$ Aufschlussgut. In einer solch geringen Menge kann mit einem Quecksilberthermometer nicht sicher genug gemessen werden. Da die Aufschlussbrenner nicht mit Thermostaten, sondern mit Energiereglern versehen sind, würde die Messung in einem grösseren Volumen (bei gleicher Einstellung des Reglers) einen falschen Wert liefern. Wir haben deshalb versucht, die Temperatur direkt in der zum Aufschluss verwendeten Menge von $1 \mathrm{ml}$ Schwefelsäure zu messen, was sich mit Hilfe eines NTCWiderstandes (oder Thermistors) befriedigend ausführen lässt. Wir verwenden dazu einen NTC-Widerstand in Miniaturausführung, Type E $205 \mathrm{CE} / \mathrm{P} 47 \mathrm{~K}$, von Philips. Der eigentliche Thermistor, eine Halbleitermaterialperle von weniger als $1 \mathrm{~mm}^{3}$ Volumen, ist in der Spitze eines feinen, $30 \mathrm{~mm}$ langen Glasröhrchens eingeschmolzen und damit auch in heisser Schwefelsäure verwendbar. Dieser Thermistor, dessen Anschlussdrähte vorher verlängert wurden, wird an ein Rohr aus Bleiglas angeschmolzen; die isolierten Drähte werden durch dieses Rohr nach aussen geführt, am Rohrende umgebogen und mit Klebstreifen fixiert. Durch Vergleich mit einem Hg-Thermometer wird sodann der elektrische Widerstand des Thermistors bei verschiedenen Temperaturen zwischen $200^{\circ} \mathrm{C}$ und $300^{\circ} \mathrm{C}$ durchgemessen, wozu ein einfaches Ohmmeter ausreicht. Widerstand bei $20^{\circ} \mathrm{C}$ etwa $47 \mathrm{k} \Omega$, bei $260^{\circ} \mathrm{C}$ etwa $200 \Omega$. Die Meßspannung darf nicht mehr als $5 \mathrm{~V}$ betragen, da sich sonst der Thermi- 
stor aufheizt und einen falschen Widerstandswert ergibt. Auf diese Weise erhält man eine Eichkurve, die es nachher erlaubt, auch in einem sehr geringen Volumen Schwefelsäure die Temperatur genau zu messen. Die Einstellung der Infrabrenner ist nach einigen Wochen zu überprüfen.

Die Neutralisation der Schwefelsäure erfolgt mit 5prozentigem Ammoniak in einem kleinen Überschuss, wobei anschliessend mit $1 n$-Schwefelsäure und $p$-Nitrophenol als Indikator auf pH 7 zurücktitriert wird. Damit wird bezweckt, dass alle Proben dieselben Ammonium- und Sulfationenkonzentration besitzen, ein Kriterium, das für die anschliessende Farbreaktion besondere Bedeutung hat [6].

Ist die nötige Ausrüstung mit Infrarotbrennern vorhanden, so gestattet diese Aufschlussmethode ein rasches Verarbeiten grösserer Probenserien. Aus diesem Grunde und auch wegen der sauberen Arbeitsweise haben wir uns schliesslich für diese Methode entschlossen, obwohl Vergleichsversuche mit dem "trockenen" Aufschluss ergeben haben, dass dieser, sehr sorgfältig ausgeführt, ebenfalls brauchbare Ergebnisse liefert. Während frühere Versuche Verluste bis zu 25\% ergaben, müssen wir heute auf Grund der bisher gemachten Erfahrungen annehmen, dass es sich hier um Adsorptionsverluste bei der kolorimetrischen Bestimmung gehandelt hat. Wie Tabelle 1 zeigt, liefert der trockene Aufschluss (Veraschung des Trockenrückstandes in der Platinschale) Resultate, welche zwar untereinander nicht in jedem Fall richtig sein können, dann nämlich, wenn der Gesamtphosphorgehalt im Filtrat grösser ist als in der unfiltrierten Probe. Gegenüber den Werten des nassen Aufschlusses aber zeigen sie doch nur geringe Unterschiede.

Tabelle 1

Bestimmung des Phosphat- und des Gesamt-P-Gehaltes in Wasserproben aus dem Untersee (Höhe Berlingen) vom 13. Oktober 1964. Die filtrierten und unfiltrierten Proben zur Gesamt-P-Bestimmung wurden trochen (Veraschung in der Platinschale) und nass (Schwefelsäuve und Perhydrol) aufgeschlossen

\begin{tabular}{|c|c|c|c|c|c|}
\hline \multirow[t]{2}{*}{$\begin{array}{l}\text { Wassertiefe } \\
(\mathrm{m})\end{array}$} & \multirow[t]{2}{*}{$\begin{array}{l}\mathrm{PO}_{4}-\mathrm{P} \\
\mu \mathrm{g} / \mathrm{l}\end{array}$} & \multicolumn{2}{|c|}{$\begin{array}{l}\text { Gesamt-P trocken } \\
\text { aufgeschlossen, } \mu \mathrm{g} / \mathrm{P}\end{array}$} & \multicolumn{2}{|c|}{$\begin{array}{l}\text { Gesamt-P nass } \\
\text { aufgeschlossen, } \mu \mathrm{g} / 1 \mathrm{P}\end{array}$} \\
\hline & & filtriert & unfiltriert & filtriert & unfiltriert \\
\hline 0 & 6,0 & 14 & 25 & 15 & 28 \\
\hline 5 & 6,0 & 15 & 26 & 14 & 29 \\
\hline 10 & 6,0 & 13 & 24 & 14 & 24 \\
\hline 15 & 7,5 & 13 & 25 & 14 & 24 \\
\hline 20 & 34,5 & 39 & 51 & 40 & 46 \\
\hline 30 & 142 & 146 & 154 & 143 & 148 \\
\hline 40 & 160 & 170 & 168 & 161 & 164 \\
\hline 43 & 169 & 190 & 184 & 170 & 175 \\
\hline
\end{tabular}

\section{Praktische Erprobung der Methode}

\section{Die zeitliche Entwicklung der Farbintensität}

Um die Frage abzuklären, ob die gegenüber der Phosphatbestimmung [1] veränderten Zusätze im Reaktionsgemisch, insbesondere Säure und Ammoniak, den zeitlichen Verlauf der Farbentwicklung beeinflussen, wurde eine Seewasserprobe aufgeschlossen und darin der Gesamtphosphorgehalt bestimmt. Die Entwicklung des 
Molybdänblaus wurde während zwei Stunden verfolgt, wobei die Messküvette, um die Probe nicht dauernd dem Lichtstrahl des Photometers auszusetzen, zwischen den Messungen aus dem Gerät entfernt wurde. Die Messwerte sind in Tabelle 2 zusammengestellt und in Abb. 1 graphisch aufgetragen.

\section{Tabelle 2}

Zeitlicher Verlauf der Ausbildung des Molybdänblaus bei der Phosphorbestimmung in einer mit Schwefelsäure aufgeschlossenen Probe

\begin{tabular}{cl}
\hline $\begin{array}{l}\text { Standzeit } \\
(\mathrm{min})\end{array}$ & $\begin{array}{l}\text { Extinktion } \\
(100 \mathrm{~mm}, 720 \mathrm{~m} \mu)\end{array}$ \\
\hline 0 & 0,075 \\
10 & 0,159 \\
20 & 0,159 \\
30 & 0,164 \\
40 & 0,164 \\
60 & 0,168 \\
80 & 0,161 \\
100 & 0,155 \\
120 & 0,145 \\
\hline
\end{tabular}

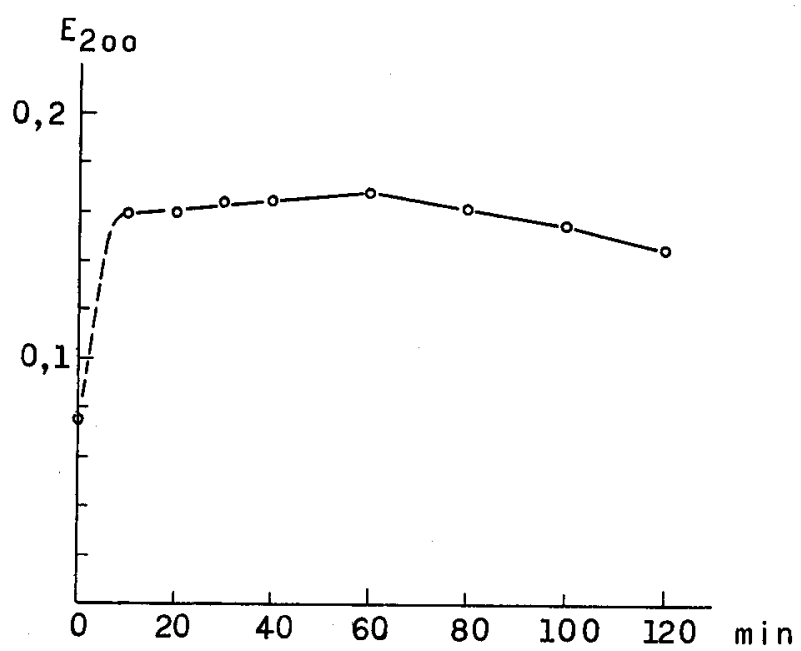

Abbildung 1

Graphische Darstellung des zeitlichen Verlaufs der Molybdänblaureaktion bei der Phosploorbestimmung in einer mit Schwefelsäure aufgeschlossenen Probe

Danach hat die Extinktion nach 30 min beinahe ihr Maximum erreicht und hält dieses während gut $40 \mathrm{~min}$. Die Proben können somit $30 \mathrm{~min}$ nach dem Ansetzen gemessen werden.

\section{Die Vollständigkeit des Aufschlusses}

Um zu prüfen, ob der Aufschluss unter den angegebenen Bedingungen vollständig verläuft, wurde aus Adenosin-5-mono-phosphorsäuremonohydrat (puriss., Rein- 
heit $\geqslant 99,5 \%$ ) eine Standardlösung hergestellt und diese aufgeschlossen. Die gefundenen Werte sind in Tabelle 3 zusammengestellt.

Tabelle 3

Resultate von A ufschlussversuchen mit Adenosin-5-mono-phosphorsäure-monohydrat (Aufschluss mit Schwefelsäure und Perhydrol)

\begin{tabular}{lll}
$\begin{array}{l}\text { Sollwert } \\
\mu \mathrm{g} / \mathrm{l} \mathrm{P}\end{array}$ & $\begin{array}{l}\text { Gefunden } \\
\mu \mathrm{g} / 1 \mathrm{P}\end{array}$ & $\begin{array}{l}\text { Gefunden } \\
\% \text { des Sollwertes }\end{array}$ \\
\hline $\mathbf{5 0}$ & 47,5 & 95 \\
$\mathbf{5 0}$ & $\mathbf{4 8}$ & 96 \\
50 & 49 & 98 \\
20 & 21 & 105 \\
20 & 20,5 & 103 \\
20 & 21 & 105 \\
\hline
\end{tabular}

Sie zeigen, dass eine Aufschlusszeit von $30 \mathrm{~min}$ bei $260^{\circ} \mathrm{C}$ genügt, um den grössten Teil der verwendeten Phosphorverbindung in anorganisches Phosphat überzuführen. $\mathrm{Da}$ es sich bei den im Seewasser gelöst oder geformt vorhandenen Phosphorkörpern um leicht zersetzliches Material handelt, darf angenommen werden, dass auch dort der Aufschluss vollständig ist.

\section{Streuungsversuch}

Zur Ermittlung der Bestimmungsgenauigkeit wurde je eine Reihe von filtrierten und unfiltrierten identischen Wasserproben aus dem Vierwaldstättersee aufgeschlossen und anschliessend ihr Phosphorgehalt bestimmt. In Tabelle 4 sind die Ergebnisse der Bestimmungen im filtrierten Seewasser zusammengestellt, in Tabelle 5 diejenigen im unfiltrierten Wasser.

Tabelle 4

Resultate eines Streuungsversuchs mit 13 identischen filtrievten Seewasserproben aus dem Vierzaldstättersee.

$\begin{array}{lcc}3,4 \mu \mathrm{g} / \mathrm{lP} & 3,0 \mu \mathrm{g} / \mathrm{lP} & 2,8 \mu \mathrm{g} / \mathrm{lP} \\ 3,2 & 3,6 & 3,2 \\ 3,2 & 3,0 & 3,2 \\ 2,8 & 3,4 & 3,6 \\ 3,0 & & \\ \text { Arithmetisches Mittel }: \bar{x}=3,2 \mu \mathrm{g} / 1 \mathrm{P} & \end{array}$

Tabelle 5

Resultate eines Streunngsversuchs mit 12 identischen unfiltrierten Seevasserproben aus dem Vierwaldstättersee

$\begin{array}{lcc}13,2 \mu \mathrm{g} / \mathrm{lP} & 13,0 \mu \mathrm{g} / \mathrm{lP} & 13,6 \mu \mathrm{g} / 1 \mathrm{P} \\ 12,8 & 13,4 & 14,6 \\ 13,4 & 12,8 & 13,6 \\ 12,4 & 13,0 & 12,8 \\ \text { Arithmetisches Mittel }: \bar{x}=13,2 \mu \mathrm{g} / \mathrm{P} & \end{array}$


Die quadratische Abweichung $s$, berechnet nach

$$
s=\sqrt{\frac{\sum\left(x_{i}-\bar{x}\right)^{2}}{N-1}},
$$

beträgt für die filtrierten Proben: $s= \pm 0,3 \mu \mathrm{g} / \mathrm{l} \mathrm{P}$,

$$
\text { wobei } \begin{aligned}
x_{i} & =\text { einzelnes Resultat, } \\
\bar{x} & =\text { arithmetisches Mittel, } \\
N & =\text { Anzahl Messwerte. }
\end{aligned}
$$

Für die unfiltrierten Proben beträgt die quadratische Abweichung $s= \pm 0,6 \mu \mathrm{g} / \mathrm{l} \mathbf{P}$.

Es ist demnach möglich, den Gesamtphosphorgehalt in filtriertem Seewasser mit einer mittleren quadratischen Abweichung von $s= \pm 0,3 \mu \mathrm{g} / \mathrm{P}$ und in unfiltriertem Wasser mit $s= \pm 0,6 \mu \mathrm{g} / \mathrm{l} \mathrm{P}$ zu bestimmen. Die etwas grössere Streuung im unfiltrierten Wasser stammt vermutlich von der ungleichmässigen Verteilung des Planktons in der Probe, insbesondere der grösseren Plankter.

Zur Filtration werden vorgereinigte, das heisst mit bidestilliertem Wasser vorgespülte Millipore-Membranfilter Type HAWP 04700 verwendet. Die Porenweite dieser Filter beträgt $0,45 \mu$. RIGLER [7], der ebenfalls diese Filter verwendet, hat indessen festgestellt, dass der Anteil des gelösten organischen Phosphors am Gesamtphosphor bei Verwendung geringerer Porenweiten zurückgeht, und vermutet, dass entweder die Filtration mit Filtern der angegebenen Type unvollständig ist oder dass bei Filtern geringerer Porenweite ein Teil der gelösten organischen Stoffe am Filtermaterial adsorbiert wird. Jedenfalls muss diese Frage weiterverfolgt werden.

\section{Geräte}

\section{Analysenvorschrift}

Sämtliche Geräte, die zur Phosphatbestimmung [1] notwendig sind. Dazu folgendes Material:

Elektrische Aufschlussbrenner (Salvis-Infrabrenner)

2 Messkolben $100 \mathrm{ml}$

Messzylinder $100 \mathrm{ml}$

Kjeldahlkolben $200 \mathrm{ml}$, Jena G 20

Siedestäbchen

\section{Reagenzien}

Bidestilliertes, filtriertes Wasser

Reagens I: Molybdän-Wolfram-Reagens, siehe [1]

Reagens II : Zinnchloridlösung, siehe [1]

Ammoniaklösung 5\%, durch Verdünnen von konzentriertem analysenreinem

Ammoniak hergestellt

Wasserstoffsuperoxyd 30\% (Perhydrol Merck)

Schwefelsäure konzentriert, analysenrein

Schwefelsäure $1 n$

$p$-Nitrophenol, 1prozentige alkoholische Lösung

Zitronensäure, 2prozentige wässrige Lösung. Jedesmal frisch zubereiten!

Phosphat-Typlösung $1 \mathrm{ml} \bumpeq 1 \mathrm{mg} \mathrm{P}$, siehe [1]. 
Arbeitsvorschrift

Die Proben sollen möglichst bald nach der Probenahme aufgeschlossen werden.

Aufschluss. $100 \mathrm{ml}$ aufgeschütteltes oder filtriertes Seewasser werden in einem Kjeldahlkolben mit $1,0 \mathrm{ml}$ konzentrierter Schwefelsäure versetzt und auf dem Infrabrenner bei Höchsttemperatur abgedampft. Vorsicht: Beim ersten Auftreten von $\mathrm{SO}_{3}$-Dämpfen Kolben wegstellen! Nach dem Erkalten 3 Tropfen Perhydrol zugeben und auf einem zweiten Infrabrenner während $30 \mathrm{~min}$ auf $260^{\circ} \mathrm{C}$ halten (Infrabrenner vorher mit Elektrothermometer eichen). Nach der Zugabe des Perhydrols tritt eine lebhafte Oxydationsreaktion ein. Anschliessend erkalten lassen, nochmals mit $10 \mathrm{ml}$ bidestilliertem Wasser aufnehmen und, um noch verbliebene Spuren von $\mathrm{H}_{2} \mathrm{O}_{2}$ zu zerstören, dieses wieder eindampfen. Die Proben sind wiederum beim ersten Auftreten von $\mathrm{SO}_{3}$-Dämpfen wegzustellen.

Neutralisation. Die so erhaltene hochkonzentrierte Schwefelsäurelösung wird mit etwas bidestilliertem Wasser verdünnt und mit 2 Tropfen $p$-Nitrophenol sowie $13 \mathrm{ml}$ 5prozentigem Ammoniak versetzt (diese Menge ist der ursprünglich zugesetzten konzentrierten Schwefelsäure unter Einrechnung eines geringen Überschusses äquivalent). Die schwach alkalische Probe wird nun sofort mit $1 n$-Schwefelsäure bis zum Neutralpunkt zurücktitriert und im Messzylinder auf $100 \mathrm{ml}$ verdünnt. Dieses Vorgehen bezweckt, dass alle Proben dieselbe Ammonium- und Sulfationenkonzentration besitzen.

Ansetzen der Typreihe. 1,00 ml Typlösung $(1 \mathrm{ml} \hat{=} 1 \mathrm{mg} \mathrm{P})$ wird im Messkolben mit bidestilliertem Wasser auf $100 \mathrm{ml}$ aufgefüllt. Davon $10 \mathrm{ml}$ in den zweiten Messkolben abpipettieren und auf $100 \mathrm{ml}$ auffüllen. Nun werden, der Typenzahl entsprechend, Erlenmeyerkolben mit etwa $50 \mathrm{ml}$ bidestilliertem Wasser, 1,0 $\mathrm{ml}$ konzentrierter Schwefelsäure, 2 Tropfen $p$-Nitrophenol und $13 \mathrm{ml} 5$ prozentigem Ammoniak versetzt und mit $1 n$-Schwefelsäure auf den Neutralpunkt zurücktitriert, worauf man sie im Messzylinder auf $100 \mathrm{ml}$ minus das Volumen der zuzusetzenden Typlösung auffüllt und schliesslich diese selber beifügt.

Folgende Typenreihe hat sich als zweckmässig erwiesen:

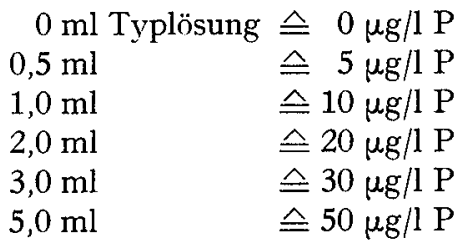

Zum Abmessen der Typlösung soll immer die gleiche Pipette verwendet werden. Für kleine P-Mengen ist es wichtig, eine Nullprobe anzusetzen.

Zugabe der Reagenzien. Reagens II im Mischzylinder verdünnen (1 Teil +9 Teile bidestilliertes Wasser). $\mathrm{Zu}$ den Proben nacheinander in rascher Folge und unter jeweiligem Schwenken genau je 1,00 ml Zitronensäure 2\%,2,00 ml Reagens I, 0,50 ml verdünntes Reagens II zugeben.

Messung im Photometer. Es gelten die für die Phosphatbestimmung angegebenen Weisungen [1]. Die Messung kann nach $20 \mathrm{~min}$ Standzeit beginnen und soll nach einer Stunde beendet sein. Da beim Aufschluss Gips $\left(\mathrm{CaSO}_{4}\right)$ und $\mathrm{SiO}_{2}$ anfallen können und 
somit immer ein kleiner, körniger Bodensatz vorhanden ist, muss die Lösung äusserst vorsichtig aus dem Kolben in die Küvette dekantiert werden.

Verdiunnen der Proben. Liegt der Phosphorgehalt der Probe zu hoch, so wird eine entsprechend geringere, aliquote Menge in den Aufschlusskolben eingemessen und die ganze Prozedur wiederholt. Der Verdünnungsgrad ist beim Auswerten zu berücksichtigen.

Angabe der Resultate. Auf $1 \mu \mathrm{g} / \mathrm{l}$ auf- oder abrunden.

\section{Zusammenfassung}

Für die serienmässige Mikrobestimmung von organisch gebundenem Phosphor in Seewasser wurde die bekannte Methode des Aufschlusses mit Schwefelsäure überprüft und modifiziert. Die wichtigste Fehlerquelle in den bisherigen Verfahren bilden die Verluste an Phosphor, welche beim Abrauchen der Schwefelsäure entstehen. Es hat sich als nötig erwiesen, sowohl die Aufschlusszeit als auch die Aufschlusstemperatur zu standardisieren.

Der Phosphatgehalt der aufgeschlossenen Probe wird sodann nach unserer Modifikation der Molybdänblaumethode [1] kolorimetrisch gemessen. Die mittlere quadratische Abweichung beträgt bei unfiltriertem Seewasser $\pm 0,6 \mu \mathrm{g} / \mathrm{l} \mathrm{P}$, bei filtriertem $\pm 0,3 \mu \mathrm{g} / \mathrm{l} \mathrm{P}$.

\section{Summary}

For the micro-analysis in series of organically bound phosphorus in lake water, the well-known method of decomposition with sulfuric acid was revised and modified. The main source of errors in the former method was caused by the loss of phosphorus during the evaporation of sulphuric acid. It was therefore found necessary to standardize the time as well as the temperature of the decomposition process.

The phosphate content of the dissolved sample is then measured colorimetrically by means of the molybdenum-blue method modified by us [1]. The mean standard deviation amounts to $\pm 0.6 \mu \mathrm{g} / \mathrm{l} P$ for not filtered lake-water and to $\pm 0.3 \mu \mathrm{g} / \mathrm{l} \mathrm{P}$ for filtered lake-water.

\section{LITERATURVERZEICHNIS}

[1] АмвӥнL, H., und SснміD, M., Die Bestimmung geringster Mengen von Phosphation im Wasser von Binnenseen, Schweiz. Z. Hydrol. 27, 172(1965).

[2] Van Beneden, G., Application d'un microdosage colorimétrique de phosphore aux eaux, Bull. CBEDE 35, 33 (1957).

[3] Börtcher, C. F. J., A rapid and sensitive submicro phosphorus determination, Anal. chim. Acta 24, 203 (1961).

[4] Burton, J. D., und Riley, J. P., Determination of soluble phosphate and total P in sea water and of total $\mathrm{P}$ on marine muds, Microchim. Acta, 1350 (1956).

[5] Kapplin, V. T., und Semenov, A., Die Zerstörung von organischen Stoffen zur Phosphor- und Stickstoffbestimmung in Fluss- und Seewasser, Jzv. Akad. Nauk SSSR, Otd. chim. 1526 (1959). Aus: Lit. Ber. 9, Nr. 723 (1960).

[6] OHLE, W., Zur Vervollkommnung der hydrochemischen Analyse, III, Die Phosphorbestimmung, $Z$. angew. Chemie 10,906 (1936).

[7] RIGLER, F. H., The phosphorus fractions and the turnover time of inorganic phosphorus in different types of lakes, Limnology and Oceanography 9, 511 (1964).

[8] WatT, W. D., und Hayes, F. R., Tracer study of the phosphorus cycle in sea water, Limnology and Oceanography 8, 276 (1963). 